\title{
Broad-line region configuration of the supermassive binary black hole candidate PG1302-102 in the relativistic Doppler boosting scenario
}

\author{
Zihao Song ${ }^{1,2}$, Junqiang $\mathrm{Ge}^{1}$, Youjun $\mathrm{Lu}^{1,2, \dagger}$, Changshuo Yan ${ }^{1,2}$, and and Xiang $\mathrm{Ji}^{1,2}$ \\ ${ }^{1}$ CAS Key Laboratory for Computational Astrophysics, National Astronomical Observatories, Chinese Academy of Sciences, No. \\ 20A Datun Road, Beijing 100101, China; †luyj@nao.cas.cn \\ 2 School of Astronomy and Space Science, University of Chinese Academy of Sciences, No. 19A Yuquan Road, Beijing, 100049, \\ China
}

November 18, 2020

\begin{abstract}
PG1302-102 is thought to be a supermassive binary black hole (BBH) system according to the periodical variations of its optical and UV photometry, which may be interpreted as being due to the relativistic Doppler boosting of the emission mainly from the disk around the secondary black hole (BH) modulated by its orbital motion. In this paper, we investigate several broad emission lines of PG1302-102 using archived UV spectra obtained by IUE, GALEX, and Hubble, to reveal the broad-line region (BLR) emission properties of this BBH system under the Doppler boosting scenario. We find that the broad lines Ly $\alpha, \mathrm{N} v, \mathrm{C}_{\mathrm{IV}}$, and $\mathrm{C}$ III] all show Gaussian profiles, and none of these lines exhibits obvious periodical variation. Adopting a simple model for the BLR, we perform Markov chain Monte Carlo fittings to these broad lines, and find that the BLR must be viewed at an orientation angle of $\sim 33^{\circ}$, close to face-on. If the Doppler boosting interpretation is correct, then the BLR is misaligned with the BBH orbital plane by an angle of $\sim 51^{\circ}$, which suggests that the Doppler boosted continuum variation has little effect on the broad-line emission and thus does not lead to periodical line variation. We further discuss the possible implications for such a BLR configuration with respect to the $\mathrm{BBH}$ orbital plane.
\end{abstract}

Key words. line: profiles-galaxies: active-galaxies: quasars: supermassive black holes: individual-PG1302-102

\section{Introduction}

Supermassive binary black holes (BBHs) are predicted in many galactic centers (e.g., Begelman et al. 1980; Chen et al. 2020) since larger galaxies are formed by hierarchical mergers of smaller ones (e.g., Cole et al. 2000; Haehnelt, \& Kauffmann 2002; Somerville \& Davé 2015), and most galaxies host a supermassive black hole (SMBH) in their centers (e.g., Magorrian et al. 1998; Kormendy \& Ho 2013). However, it is hard to directly detect BBH systems at subparsec separations because of the limitation in spatial resolutions of current available facilities (e.g., Yu 2002). BBH systems must be selected because of some particular signatures, such as orbital modulated periodical light curves (e.g., Graham et al. 2015a,b; Charisi et al. 2016, 2018; Li et al. 2019), double-peaked or asymmetric line pro-files (e.g., Tsalmantza et al. 2011; Eracleous et al. 2012; Ju et al. 2013; Liu et al. 2014; Guo et al. 2019), deficiency of optical-UV continuum radiation (e.g., Yan et al. 2015; Zheng et al. 2016), and broad line polarization (Savić et al. 2019). More than 100 BBH candidates have been proposed in the literature according to various observational signatures, and most of them are based on the periodicity in their optical light curves, (e.g., Graham et al. 2015b; Charisi et al. 2016, 2018; Liu et al. 2019; Chen et al. 2020; Liao et al. 2020).

One of the most intriguing BBH candidates is PG1302-102 at redshift $z=0.2784$, which was monitored for more than 20 years. Its optical and UV light curves show clear periodical variations with a period of $\sim 1884$ days in the observer's rest frame, corresponding to $\sim 1474$ days in the PG1302-102 rest frame (Graham et al. 2015a). The sinusoid shape of its light curves can be well fitted by orbital modulated Doppler boosted emission from the disk around the secondary black hole (BH) in an unequal BBH system (D'Orazio et al. 2015). In this scenario, the BBH orbital plane must be viewed at an orientation angle, defined as the angle between the line of sight (LOS) and the normal direction of the BBH orbital plane, $i_{\text {orb }} \geq 60^{\circ}$, close to being viewed edge-on, in order to explain the amplitude ( 14\%) of the optical variability of PG1302-102, with a spectral index of $\alpha_{\mathrm{opt}}=1.1$ (D'Orazio et al. 2015; Xin et al. 2020).

For a BBH system like that proposed for PG 1302-102, if its broad-line region (BLR) is flattened and aligned with the BBH orbital plane and also viewed at an orientation close to edgeon (D'Orazio et al. 2015), then the broad emission lines emitted from it are expected to be double-peaked (e.g., Liu et al. 2014; Nguyen \& Bogdanović 2016; Nguyen et al. 2019). The broadline profiles are also expected to vary periodically because the BLR clouds receive Doppler boosted and attenuated ionizing flux periodically (see Ji et al. 2020). However, whether the BLR is aligned with the $\mathrm{BBH}$ orbital plane for $\mathrm{BBH}$ systems is not clear. For a circumbinary BLR with a flat disk-like structure, if it is misaligned with the edge-on viewed BBH orbital plane, the asymmetry modulated by the Doppler boosting effect on broadline profiles may be weaker with increasing offset orientation angles, and the profile could also be Gaussian rather than doublepeaked. 
To investigate the properties of broad emission lines of PG 1302-102, we collect the archived spectroscopic data from the past 40 years to check whether the broad-line profiles vary in such a long time interval, and how the orientation of BLR correlates with the orbital orientation constrained under the Doppler boosting BBH scenario. In Section 2 we describe the spectral analyses and introduce a simplified BLR model for fittings of the broad emission lines. In Section 3 we analyze the profiles of some broad emission lines, and compare the model fitted BLR orientation angle with that of the $\mathrm{BBH}$ system under the Doppler boosting BBH scenario. In Section 4 we discuss possible implications. In Section 5 we summarize our main results.

\section{Data analysis and model fitting}

PG 1302-102 has been photometrically monitored for a long time in the optical and UV bands. Graham et al. (2015a) first discovered the periodical variation of PG1302-102 in the optical band with a period $P_{\text {orb }} \sim 1884$ days by using the Catalina Real-time Transient Survey (CRTS) data, and they suggest this variation may indicate the existence of a BBH in PG1302-102. Since then PG1302-102 has been intensively studied in the literature. D'Orazio et al. (2015) proposed that the optical and UV light curves of PG1302-102 may be explained as being due to the Doppler boosting of continuum radiation from an accretion disk associated with the secondary $\mathrm{BH}$ rotating around the primary BH in a BBH system, and they obtained $P_{\text {orb }} \sim 1996$ days. Liu et al. (2018) further considered the damped random walk (DRW) process in the analysis of the PG1302-102 light curve by including additional data from All-Sky Automated Survey for Supernovae $($ ASAS-SN $),{ }^{1}$ and they found $P_{\text {orb }} \sim 2026$ days. In this paper we adopt $P_{\text {orb }}=1996$ days, the value obtained by D'Orazio et al. (2015) using the Doppler boosting model to fit the light curves, which corresponds to $~ 1561$ days in the PG1302-102 rest frame.

\subsection{Spectral sample and data analysis}

PG1302-102 has been observed many times in optical and UV bands over the past 40 years with the International Ultraviolet Explorer (IUE) ${ }^{2}$, GALaxy Evolution EXplorer (GALEX), ${ }^{3}$ and Hubble Space Telescope (HST) ${ }^{4}$. From the archived spectral data we identify eight $\mathrm{Ly} \alpha+\mathrm{N} v$ broad emission lines (three, two, and three from IUE, GALEX, and HST, respectively), three $\mathrm{C}_{\text {III] }}$ broad emission lines (two GALEX and one HST), and three $\mathrm{C}$ Iv broad emission lines (two GALEX and one HST), whose peak fluxes are all higher than the $3 \sigma$ significance level. These spectra with broad emission lines detected actually include three components: the continuum emitted from the accretion disk, the broad emission line from the BLR, and $\mathrm{Fe}_{\text {II }}$ lines from the BLR. Therefore, we apply a model with three components to fit each observed spectrum, i.e., a power-law for continuum, a Gaussian profile for each broad line, and multiple lines from Fe II templates (Sigut \& Pradhan 2003; Sigut et al. 2004) described by Gaussian profiles with the same velocity dispersion. As done in Song et al. (2020), the wavelength windows in the rest frame for the fittings to each broad line are $950-1300 \AA, 1180-1280 \AA$, $1450-1650 \AA, 1800-2000 \AA, 4250-4650 \AA$, and $4800-5200 \AA$, and the windows for Fe II lines are $950-1195 \AA, 1250-1300 \AA$,

\footnotetext{
1 https://asas-sn.osu.edu

2 http://www.vilspa.esa.es/iue/iue.html

3 https://www.nasa.gov/centers/jpl/missions/galex.html

4 https://www.nasa.gov/mission_pages/hubble
}

$1450-1530 \AA, 1570-1650 \AA, 1890-1880 \AA$, and $1940-2000 \AA$. Three fitting examples are shown in the top row of Figure 1; the panels from left to right correspond to $\mathrm{Ly} \alpha+\mathrm{N} \mathrm{v}, \mathrm{C}_{\mathrm{IV}}$, and C III] broad emission lines.

For an active SMBH, the BLR size can be inferred from its optical luminosity by using the empirical relationship between BLR size and optical luminosity as (Bentz et al. 2013)

$\log \left(R_{\mathrm{BLR}}^{L} /\right.$ lt-days $) \simeq 1.527+0.533 \log \left[\frac{\lambda L_{\lambda}(5100 \AA)}{10^{44} \mathrm{erg} \mathrm{s}^{-1}}\right]$.

The above relationship may also be valid in the case of an active $\mathrm{BBH}$ system if the BLR is far away from the $\mathrm{BBH}^{5}$ since the broad-line emission is determined by the photonionization processes (e.g., Korista \& Goad 2000). According to the spectra from the KPNO 2.1 telescope and Gold Spectrograph (Boroson \& Green 1992), we have $\lambda L_{\lambda}(5100 \AA) \sim 3.2 \times$ $10^{45} \mathrm{erg} \mathrm{s}^{-1}$, thus $R_{\mathrm{BLR}} \sim 213$ light-days (lt-days). The FWHM of the $\mathrm{C}_{\mathrm{IV}}$ is $5089 \pm 885 \mathrm{~km} \mathrm{~s}^{-1}$ according to our fittings to this line, $\lambda L_{\lambda}(1350 \AA) \sim 7.8 \times 10^{45} \mathrm{erg} \mathrm{s}^{-1}$, then the central mass of the system should be $\sim 10^{9.1} M_{\odot}$, according to the empirical mass estimator based on $\mathrm{C}_{\mathrm{IV}}$ given in Vestergaard \& Peterson (2006), which is consistent with that estimated by Graham et al. (2015a), i.e., $\sim 10^{8.3}-10^{9.4} M_{\odot}$, calculated by combining the empirical virial mass (Shen et al. 2011) and that inferred from the damped random walk (DRW) variability (MacLeod et al. 2010).

The separation of the BBH system can be estimated if the total mass $\left(M_{\bullet \bullet}\right)$ and the orbital period $\left(P_{\text {orb }}\right)$ of the BBH system are known, and it can be given by

$a_{\mathrm{BBH}}=16.4 \times\left(\frac{M_{\bullet \bullet}}{10^{9.1} M_{\odot}}\right)^{1 / 3}\left(\frac{(1+z) P_{\text {orb }}}{1996 \text { days }}\right)^{2 / 3}$ lt-days.

Adopting the observed orbital period $P_{\mathrm{obs}}=(1+z) P_{\mathrm{orb}}=1996$ days obtained from the Doppler boosting based light curve modeling (D'Orazio et al. 2015) and the estimated mass $\sim 10^{9.1} M_{\odot}$, we have $a_{\mathrm{BBH}} \sim 16.4$ lt-days. If we adopt the mass range given in (D'Orazio et al. 2015), we have $a_{\mathrm{BBH}} \sim 8.8-20.6$ lt-days. If we adopt the Doppler boosting hypothesis to model the periodical light curves of PG1302-102, $M_{\bullet \bullet}$ is required to be $>10^{9.1} M_{\odot}$ (see also Fig. 3), and thus $a_{\mathrm{BBH}} \sim 16-21 \mathrm{lt}$-days. According to all the above estimates, the ratio of the size of the BLR to the separation of the $\mathrm{BBH}$ is $R_{\mathrm{BLR}}^{\mathrm{L}} / a_{\mathrm{BBH}} \sim 10-24$, which indicates that the BLR size of PG1302-102 is indeed much larger than the $\mathrm{BBH}$ separation, and thus it is reasonable to assume a circumbinary BLR for PG1302-102 as we do in our modeling of the BLR region.

\subsection{Simple model for broad-line fitting}

The broad emission line profiles are determined by the BLR geometry, kinematics, and structures. The BLR geometry is mainly characterized by the radial and angular distributions of BLR clouds. As described in Pancoast et al. (2014a,b), the radial distribution of BLR clouds can be described as a shifted $\Gamma$-distribution, $r=R_{\mathrm{S}}+R_{\mathrm{BLR}} F+\beta^{2} R_{\mathrm{BLR}}(1-F) g$, where $R_{\mathrm{S}}=$ $2 G M_{\bullet \bullet} / c^{2}$ is the Schwarzschild radius, $R_{\mathrm{BLR}}$ is the mean value of the shifted $\Gamma$-distribution, $F=R_{\text {in }} / R_{\mathrm{BLR}}, R_{\text {in }}$ is the inner radius of BLR, and $\beta$ is the shape parameter of $\Gamma$-distribution $g\left(\beta^{-2}, 1\right)$. The angle displacement of the BLR clouds is given by $\theta=\arccos \left(\cos \theta_{0}+\left(1-\cos \theta_{0} \times U^{\gamma}\right)\right.$, where $\theta_{0}$ is the

\footnotetext{
5 As demonstrated below, for the case of PG1302-102 the estimated BLR size is indeed significantly larger than the $\mathrm{BBH}$ semimajor axis.
} 

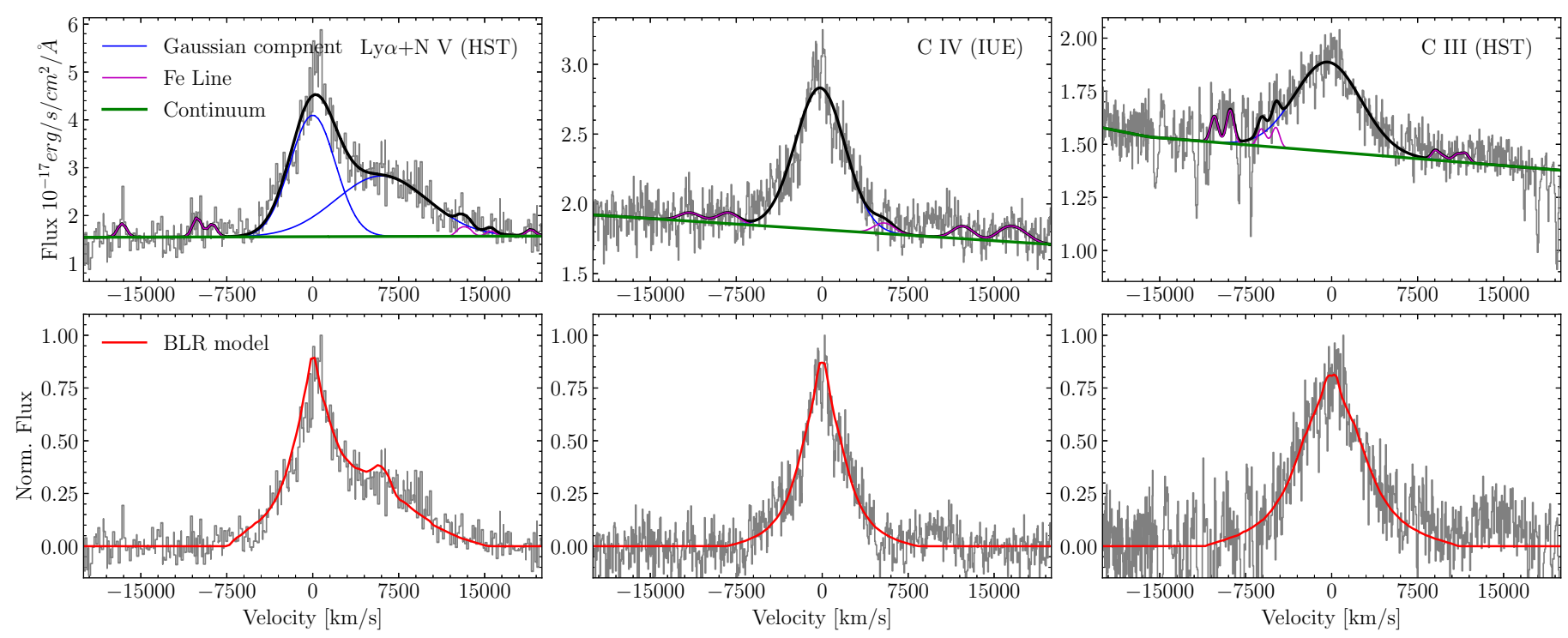

Fig. 1. Examples of the spectral decomposition (top panels) and the model fitting (bottom panels) to the broad emission lines Ly $\alpha+\mathrm{N} v$ (left), $\mathrm{C}$ IV (middle), and C III] (right). In the top panels the observed spectra (gray curves) are fitted by three components, i.e., a power law continuum (green), a Gaussian broad-line component (blue, for $\mathrm{Ly} \alpha+\mathrm{N} v$ we use two broad Gaussian components, with the separation of the two line peaks fixed at $6000 \mathrm{kms}^{-1}$ ), and multiple lines from the Sigut et al. (2004) Fe II templates (magenta). In the bottom panels the gray curves show the broad-line profiles with the continuum and Fe II lines subtracted. The red lines represent the best BLR model fitting results as introduced in Section 2.2.

opening angle and $U$ is uniformly distributed between 0 and 1 (Pancoast et al. 2014a,b; Gravity Collaboration et al. 2018). By adopting this simple BLR model introduced by Pancoast et al. (2014a), we assume that the BLR clouds rotate around the central $\mathrm{BBH}$ system on circular orbits, and constrain the size and structure of the BLR by the following seven model parameters, the same as those in Section 2.3 of Song et al. (2020): the mean and inner radius of $\operatorname{BLR}\left(R_{\mathrm{BLR}}, R_{\mathrm{in}}\right)$, radial shape parameter $(\beta)$, disk edge illumination parameter $(\gamma)$, opening angle $\left(\theta_{\mathrm{o}}\right)$, orientation angle $\left(i_{\mathrm{BLR}}\right)$ defined as the angle between the LOS and the normal direction of the flattened BLR middle plane, and the central SMBH mass (M.o). Here, the opening angle $\theta_{0}$ is defined as half of the angular thickness of the BLR, with $\theta_{0}=\pi / 2$ corresponding to a spherical BLR.

To fit the broad emission lines with the BLR model and derive a robust estimation to these seven parameters, we adopt the Markov chain Monte Carlo (MCMC) code "emcee" (Foreman-Mackey et al. 2013) to perform the model fitting after a series of prior setups. The prior of each parameter is set as follows. We allow the mean radius $R_{\mathrm{BLR}}$ uniformly distributed in the range $\left[0.2 R_{\mathrm{BLR}}^{L}, 5 R_{\mathrm{BLR}}^{L}\right]$, with the inner radius $R_{\mathrm{in}}$ in the range $\left[0, R_{\mathrm{BLR}}\right]$. The orientation angle $i_{\mathrm{BLR}}$ is set to range from $0^{\circ}$ (face-on) to $90^{\circ}$ (edge-on), the open angle $\theta_{\mathrm{o}}$ ranges from $0^{\circ}$ (flat disk) to $90^{\circ}$ (spherical BLR). The edge illumination parameter of the BLR disk are in the range $[1,5]$, and the total binary mass ranges from $\left[10^{8.3}\right.$ to $\left.10^{9.4} \mathrm{M}_{\odot}\right]$ (Graham et al. 2015a). The bottom panels of Figure 1 show three BLR model fitting examples of $\mathrm{Ly} \alpha+\mathrm{N}$ v(left), $\mathrm{C}$ IV (middle), and $\mathrm{C}$ III] (right) broad emission lines, each with the reduced $\chi_{v}^{2} \sim 1$.

\section{Results}

In order to investigate whether the Doppler boosting effect is reflected by features appearing in broad-line profiles, we first identify how the observed profiles vary at different times, then analyze the MCMC fitted BLR parameters and their correlations with that predicted by Doppler boosting hypothesis of the $\mathrm{BBH}$ system, and finally provide constraints on parameters of the binary system possibly existing for PG1302-102.

\subsection{Profile variations of broad emission lines}

According to the periodical light curves in optical and UV bands, PG1302-102 is believed to host a BBH system in the center and should be observed at a close to edge-on orbital orientation, which can lead to Doppler modulated periodical variations of optical and UV luminosities. Supposing that the BLR is aligned with the BBH orbital plane, the emitted broad emission lines should show some double-peaked or flat-topped features, which are characteristic signature of flattened BLR viewed at an orientation close to edge-on. If the secondary SMBH dominates the total luminosity and rotates in relativistic orbital velocity (D'Orazio et al. 2015), the broad emission line profiles should also be characterized by periodical asymmetry.

The top panels of Figure 2 show the mean line profiles obtained by stacking spectra observed at different times over the past 40 year for Ly $\alpha+\mathrm{N}$ v (left), C IV (middle), and C III] (right) broad lines. These broad lines all show Gaussian profiles and do not have any significant evidence for double-peaked, flat-topped, or asymmetric features. ${ }^{6}$ However, if the BLR is flattened and is aligned with the BBH orbital plane, the broad lines emitted from the BLR should be double-peaked when viewed at an orientation close to edge-on; if the BLR is spherically distributed, the broad lines should be flat-topped viewed at any orientation. Nondetection of such line features for PG 1302-102 suggests that the BLR is not aligned with the BBH orbital plane.

To further explore the detailed differences, we obtain the spectral residuals for each observed spectrum by subtracting the corresponding mean spectrum (black profiles in the top row of Fig. 2), which is obtained as follows. We first normalize the to-

\footnotetext{
6 The signal-to-noise ratios ( $\mathrm{S} / \mathrm{Ns}$ ) of the available spectra are not high, which may cause the smearing of such features if they exist. Future higher $\mathrm{S} / \mathrm{N}$ spectra may further check this.
} 

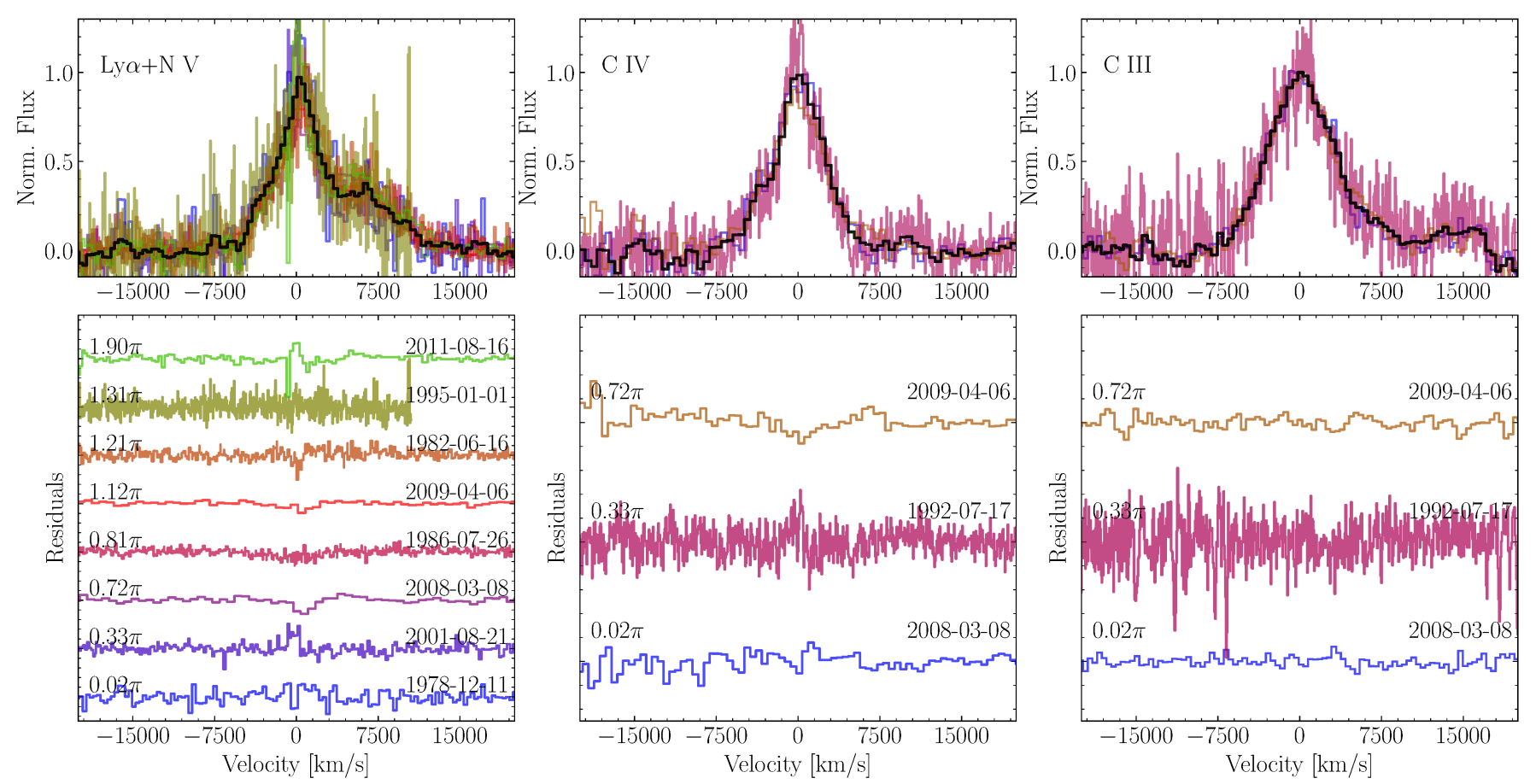

Fig. 2. Broad emission line profiles of PG1302-102 derived from IUE, GALEX, and HST observations. Columns from left to right show the Ly $\alpha+\mathrm{N}_{\text {v }}, \mathrm{C}_{\text {IV }}$, and $\mathrm{C}_{\mathrm{III}}$ broad lines. Top panels show all the observed profiles, normalized to the peak flux of mean broad-line spectrum, with the continuum and $\mathrm{Fe}$ II lines subtracted, while the bottom panels present the corresponding profile residuals of each broad line with the averaged broad emission line subtracted. For each broad-line profile and residuals, the colors from blue to green correspond to spectra observed from $\sim 40$ years ago to recent years, with the exact observation time and phase indicated above each spectrum (top right and top left, respectively) in the bottom panels.

tal flux of each broad line, then re-sample the wavelength points of each spectrum to the one with lowest spectral resolution, and finally stack all of them to derive the mean spectrum of each broad emission line. Considering that the archived spectra are observed by different instruments, we hence interpolate the mean spectrum of each broad line to the same wavelength sampling as the observed ones, and then obtain the corresponding spectral residuals, as shown in the bottom row of Figure 2. For each residual spectrum we label the corresponding phase according to its observation time based on the modeling of optical light curves given by D'Orazio et al. (2015), which would help us to understand if there are any variation trends. To search for possible variation in broad-line profiles, we take the standard deviation of residual spectrum in the ranges $[-15000,-7500] \mathrm{km} / \mathrm{s}$ and $[7500,15000] \mathrm{km} / \mathrm{s}$ as the noise of the residual spectrum. These two wavelength ranges only contain iron lines and show no feature of $\mathrm{Ly} \alpha+\mathrm{N} v \mathrm{C}$ IV and C III] emissions for the current sample. Although the spectral residuals fluctuate in the velocitywavelength space, no spectra have continuing three wavelength points over $3 \sigma$ significance level. Perhaps limited by spectral $\mathrm{S} / \mathrm{N}$, the spectral residuals at all phases for the three broad emission lines show no significant asymmetric or double-peaked features that can reflect the profile variations that modulated by the Doppler boosting effect.

These results suggest that the Doppler boosting effect, if any, does not influence or modulate the broad-line emission of PG 1302-102. This can be explained if the BLR is not aligned with the BBH orbital plane, i.e., the BLR is viewed at an orientation close to face-on, significantly offset from the orbital plane. In this case the ionizing flux received by clouds in the flattened
BLR would be Doppler boosted and modulated by the motion of the projected orbital velocity of the secondary $\mathrm{BH}$, i.e., $v_{2} \cos \Delta i$ ( $\Delta i=i_{\text {orb }}-i_{\mathrm{BLR}}$ ), where $\Delta i$ is the offset angle. When $\Delta i$ is large enough, the Doppler boosting effect on the broad emission line profiles would be smaller or even negligible compared to the case with the BLR aligned with the BBH orbital plane, and thus no periodical variation of broad-line profiles would be expected. Further understanding of the BBH system in PG 1302-102 requires more detailed parameters of the BLR geometry and the information of how it is misaligned with the BBH orbital plane.

\subsection{BLR model fitting}

Similar to our previous work (Song et al. 2020), we adopt the MCMC code "emcee" to fit the $\mathrm{Ly} \alpha+\mathrm{N} \mathrm{v}, \mathrm{C}$ IV , and C III] broad lines, and obtain the best fit for the model parameters of BLR.

In the BLR model fitting, considering that $\mathrm{Ly} \alpha, \mathrm{Nv}, \mathrm{C}$ Iv, and $\mathrm{C}$ III] lines might be emitted from different BLRs (e.g., Korista \& Goad 2000), we hence set the the seven model parameters used for constructing the BLR as free parameters for the four broad lines. In addition, we also set the shift of line center and flux of the broad line as free parameters. For the joint fitting of Ly $\alpha$ and $\mathrm{N} v$ lines, we only bound the shift of line centers for the two lines in the model fitting, with the center of $\mathrm{N} \mathrm{v}$ red-shifted $6000 \mathrm{~km} / \mathrm{s}$ from the center of Ly $\alpha$. Each observed line profile can provide a probability distribution function (PDF) for each parameter. For each broad emission line, to improve the robustness of parameter estimation, we stack together all the PDFs (eight for Ly $\alpha$ and $\mathrm{N} v$, three for C IV, and three for C III]) to derive the most robust parameter estimation (see Cols. 3-6 of 


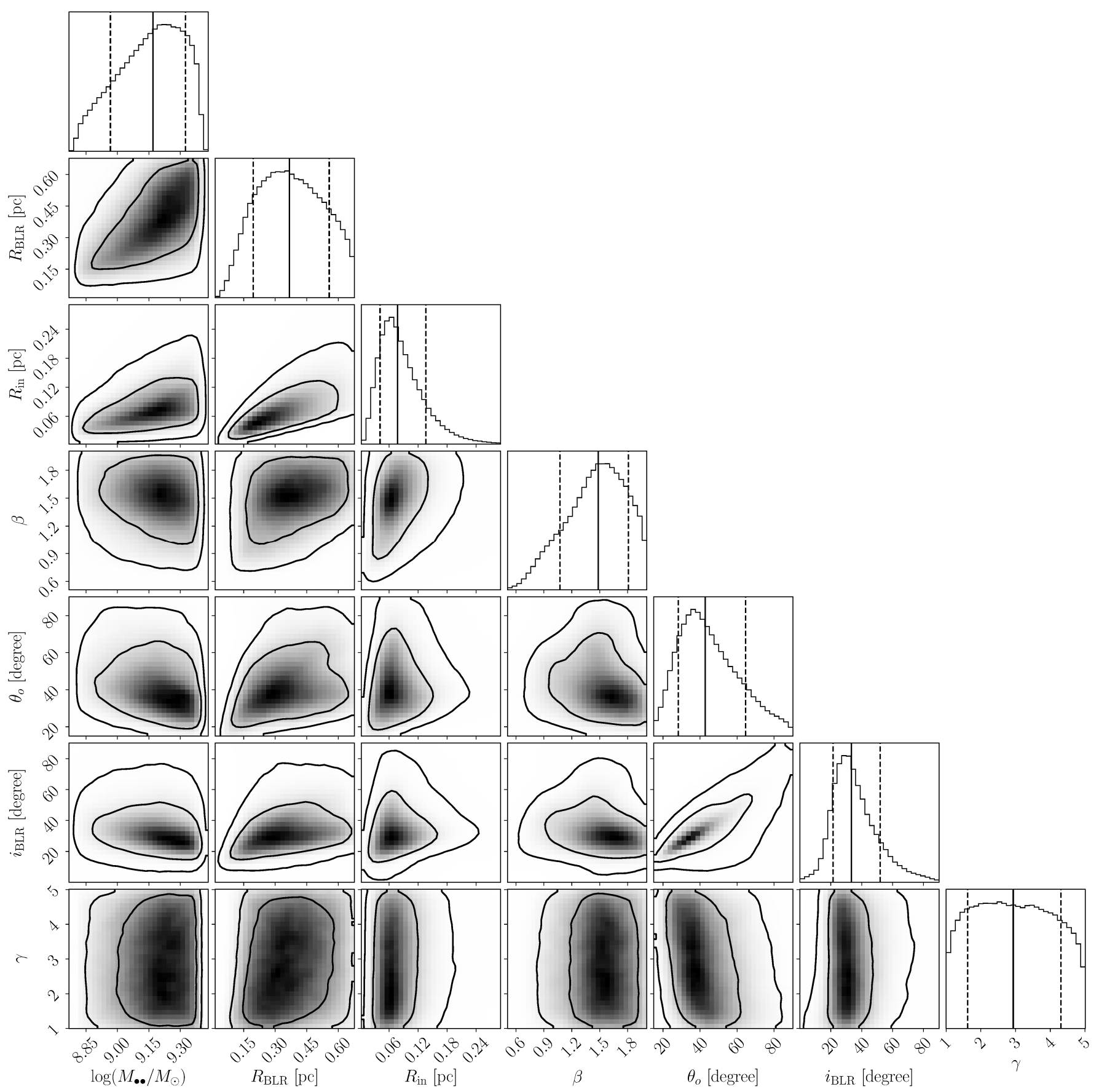

Fig. 3. Two-dimensional and one-dimensional probability distributions of the model parameters obtained from the MCMC fittings to the broad emission line profiles with a simple BLR model. The seven model parameters shown here are the total mass $\log \left(M_{\bullet \bullet}\right)$, BLR size $R_{\mathrm{BLR}}$, BLR inner radius $R_{\mathrm{In}}, \beta$, BLR opening angle $\theta_{\mathrm{o}}$, viewing angle $i_{\mathrm{BLR}}$, and $\gamma$. In the right side panels, the solid and dashed lines indicate the median, 16th, and 84th percentiles of the one-dimensional projected probability distribution function for each model parameter.

Table 1). All seven of the parameters fitted from the four broad emission lines are consistent with each other in $1 \sigma$ confidence level. Therefore, to make the parameter estimation more robust, we also stack all the PDFs derived from eight $\mathrm{Ly} \alpha+\mathrm{N} v$, three $\mathrm{C}$ IV, and three $\left.\mathrm{C}_{\mathrm{III}}\right]$ line fittings together, which produce the final values of the seven model parameters (Col. 2 of Table 1).

Figure 3 shows the stacked PDFs of all the seven parameters in our BLR model, with increasing probabilities labeled from gray to dark colors, and the two black contours enclose the
$68.3 \%$ and $95.4 \%$ of the population. For each parameter, the two black contours enclose $68.3 \%$ and $95.4 \%$ of the PDF. Table 1 lists the median value with uncertainties represented by the 16th and 84 th percentiles, which are plotted by vertical lines in the top panels of Figure 3. The MCMC fitting of broad emission lines for PG1302-102 predicts a total BH mass of $10^{9.17} M_{\odot}$ with a typical BLR radius $R_{\mathrm{BLR}} \sim 0.37_{-0.17}^{+0.19} \mathrm{pc}$. The open angle $\theta \sim 43_{-15}^{+22}$ degree and the orientation angle $i_{\mathrm{BLR}} \sim 33_{-12}^{+19}$ degree indicate that the BLR is viewed face-on instead of the edge-on view to the 
Table 1. MCMC fitted BLR model parameters for PG1302-102.

\begin{tabular}{|c|c|c|c|c|c|c|}
\hline Parameter & Mean & $\overline{\mathrm{Ly} \alpha}$ & $\overline{\mathrm{Nv}}$ & $\overline{\overline{C_{\text {IV }}}}$ & 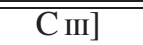 & Description \\
\hline $\log \left(M_{\bullet \bullet} / M_{\odot}\right)$ & $9.17_{-0.20}^{+0.15}$ & $9.16_{-0.19}^{+0.15}$ & $9.14_{-0.20}^{+0.17}$ & $9.17_{-0.23}^{+0.14}$ & $9.19_{-0.21}^{+0.15}$ & Total mass of BBHs \\
\hline$R_{\mathrm{BLR}}(\mathrm{pc})$ & $0.37_{-0.17}^{+0.19}$ & $0.42_{-0.18}^{+0.19}$ & $0.22_{-0.10}^{+0.18}$ & $0.35_{-0.17}^{+0.20}$ & $0.29_{-0.13}^{+0.11}$ & Mean BLR radius \\
\hline$R_{\text {in }}(\mathrm{pc})$ & $0.08_{-0.04}^{+0.06}$ & $0.09_{-0.04}^{+0.07}$ & $0.09_{-0.04}^{+0.07}$ & $0.07_{-0.03}^{+0.104}$ & $0.07_{-0.03}^{+0.05}$ & BLR Inner radius \\
\hline$\beta$ & $1.48_{-0.41}^{+0.32}$ & $1.55_{-0.28}^{+0.29}$ & $1.39_{-0.06}^{+0.43}$ & $1.54_{-0.03}^{+0.23}$ & $1.25_{-0.35}^{+0.43}$ & Radial shape parameter \\
\hline$\theta_{\mathrm{O}}\left({ }^{\circ}\right)$ & $42.8_{-14.6}^{+21.8}$ & $37.8_{-116}^{+18.1}$ & $45.2_{-16.3}^{+23.0}$ & $44.5_{-117}^{+9.80}$ & $52.6_{-16.8}^{+18.8}$ & Opening angle \\
\hline$i_{\mathrm{BLR}}\left({ }^{\circ}\right)$ & $33.3_{-11.8}^{+14.6}$ & $31.4_{-9.37}^{+11.6}$ & $31.5_{-13.2}^{+21.3}$ & $35.0_{-6.72}^{+4.7}$ & $41.2_{-162}^{-16: 2}$ & orientation angle \\
\hline$\gamma$ & $2.93_{-1.32}^{+1.39}$ & $3.17_{-1.38}^{+1.24}$ & $3.19_{-1.38}^{+1.23}$ & $2.83_{-1.26}^{+1.41}$ & $2.56_{-111}^{+1.52}$ & Edge illumination parameter \\
\hline
\end{tabular}

BBH orbital plane (e.g., D’Orazio et al. 2015; Xin et al. 2020) that is predicted by the Doppler boost hypothesis based on the optical and UV light curves.

\subsection{Misalignment between the BLR middle plane and the $\mathrm{BBH}$ orbital plane}

Figure 4 shows the total mass of BBH $M_{\bullet \bullet}$ as a function of orientation angles for both the BLR and BBH orbital planes, where we can see that the PDF of $i_{\mathrm{BLR}}$ (black shaded regions with black contours) are clearly separated from the $i_{\text {orb }}$ distributions (red shaded region), which are the same as that shown in Figure 1 of D'Orazio et al. (2015). Panels from left to right show that smaller fractions of the luminosity contribution from the secondary $\operatorname{SMBH}\left(f_{2}\right)$ actually induce larger differences between $i_{\mathrm{BLR}}$ and $i_{\text {orb }}$.

To explore the detailed difference between the misaligned $i_{\mathrm{BLR}}$ and $i_{\mathrm{orb}}$, we calculate the probability distribution of the offset between $p\left(i_{\mathrm{BLR}}, M_{\bullet \bullet}\right)$ (black shaded region in Figure 4$)$ and the PDF of BBH orbital plane constrained by the Doppler hypothesis $p\left(i_{\text {orb }}, M_{\bullet \bullet}, q, f_{2}\right)$ (red shaded region in Figure 4 ) as

$p\left(\Delta i, f_{2}\right)=$

$\iiint p\left(i_{\mathrm{BLR}}, M_{\bullet \bullet}\right) p\left(i_{\mathrm{orb}}, M_{\bullet \bullet}, q, f_{2}\right) d i_{\mathrm{orb}} d \log M_{\bullet \bullet} d q$,

where $p\left(i_{\mathrm{BLR}}, M_{\bullet \bullet}\right)=p\left(i_{\text {orb }}-\Delta i, M_{\bullet \bullet}\right)$.

Given a mass ratio $(q)$ of the $\mathrm{BBH}$, we can derive the allowed area $S\left(q, f_{2}\right)$ for the $\mathrm{BBH}$ in the $i_{\text {orb }}-\log M_{\bullet \bullet}$ plane, as shown by the top right red shaded region in Figure 4. Assuming that the probabilities for each $q$ or $f_{2}$ are the same, then the probability distribution of $M_{. \bullet}-i_{\text {orb }}$ can be defined as

$$
\begin{aligned}
p\left(i_{\text {orb }}, M_{\bullet \bullet}, q, f_{2}\right) & \propto \frac{p(q) \cdot p\left(f_{2}\right)}{p\left(S\left(q, f_{2}\right)\right) \cdot S\left(q, f_{2}\right)} \\
& \propto \frac{p\left(f_{2}\right) \cdot \frac{d S\left(q, f_{2}\right)}{d q}}{S\left(q, f_{2}\right)},
\end{aligned}
$$

where $p(q)$ and $p\left(f_{2}\right)$ are the probability distribution of the mass ratio and the luminosity fraction, and $S\left(q, f_{2}\right)$ is the allowed area for the BBH derived from the relativistic Doppler boosting limitation for the BBH system with given $q$ and $f_{2}$ as shown in Figure 4 . Since the mass ratio is not provided by any other independent method, we assume that $q$ could be any value and that $p(q)$ is uniformly distributed in the range from 0 to 1 . We also adopt a few fixed values for $f_{2}$ and $p\left(f_{2}\right) \propto \delta\left(f_{2}-C\right)$ with $C$ a constant. If adopting the mass range $\log \left(M_{\bullet \bullet} / M_{\odot}\right) \sim[8.3,9.4]$, as given in Graham et al. (2015a), we obtain

$S\left(q, f_{2}\right)=\int_{8.3}^{9.4} \int_{0}^{1} d \log M_{\bullet \bullet}\left(q, f_{2}, i_{\text {orb }}\right) d \sin i_{\text {orb }}$, and the mass of BBHs can be constrained by the optical light curves under the Doppler boosting induced periodical variation scenario as

$$
\begin{aligned}
\log \frac{M_{\bullet \bullet}}{M_{\odot}}= & 9.1+3 \log \left(\frac{c}{13214 \mathrm{~km} \mathrm{~s}^{-1}} \times \frac{\Delta L_{\mathrm{tot}}^{\mathrm{V}}}{L_{\mathrm{tot}}^{\mathrm{V}}}\right. \\
& \times \frac{1}{3-\alpha_{\mathrm{opt}}} \times \frac{1+q}{1.5} \times \frac{1}{\sin i_{\mathrm{orb}}} \\
& \left.\times \frac{1}{f_{2}-q\left(1-f_{2}\right)}\right)+\log \left(\frac{(1+z) P_{\mathrm{orb}}}{1996 \mathrm{~d}}\right),
\end{aligned}
$$

where the power-law index at the optical band $\alpha_{\mathrm{opt}}=1.1$. The total luminosity $L_{\text {tot }}^{\mathrm{V}}$ is contributed by three components, i.e., the mini-disk around the secondary $\mathrm{BH}$ and that around the primary $\mathrm{BH}$, and a circumbinary disk (CBD), hence it can be written as $L_{\text {tot }}^{\mathrm{V}}=L_{1}^{\mathrm{V}}+L_{2}^{\mathrm{V}}+L_{\mathrm{CBD}}^{\mathrm{V}}$. Considering that the primary $\mathrm{BH}$ is Doppler modulated by a line of sight velocity $v_{1} \sin i_{\text {orb }}=-q v_{2} \sin i_{\text {orb }}$, and if we assume that the contribution of CBD is constant over time (see Methods in D'Orazio et al. 2015), then we have

$$
\begin{aligned}
\frac{\Delta L_{\mathrm{tot}}^{\mathrm{V}}}{L_{\mathrm{tot}}^{\mathrm{V}}} & =\frac{\Delta L_{1}^{\mathrm{V}}+\Delta L_{2}^{\mathrm{V}}}{L_{\mathrm{tot}}^{\mathrm{V}}} \\
& =\left(3-\alpha_{\mathrm{opt}}\right) \frac{v_{2} \sin i_{\text {orb }}}{c}\left[f_{2}-q\left(1-f_{2}\right)\right] .
\end{aligned}
$$

Here the rotation velocity of the secondary $\mathrm{BH} v_{2}$ can be obtained by assuming a circular orbit for the $\mathrm{BBH}$ system, as done in D'Orazio et al. (2015):

$v_{2}=13214\left(\frac{1.5}{1+q}\right)\left(\frac{M_{\bullet \bullet}}{10^{9.1} M_{\odot}}\right)^{\frac{1}{3}}\left(\frac{(1+z) P_{\text {orb }}}{1996 \text { day }}\right)^{-\frac{1}{3}} \mathrm{kms}^{-1}$.

Figure 5 shows the probability distribution of the orientation angle offset $\Delta i$ in the case of $f_{2}=1.0,0.95$, and 0.9 . For the luminosity fraction of the secondary $\mathrm{BH} f_{2}$ decreasing from 1.0 to 0.9 , the offset between $i_{\text {orb }}$ and $i_{\mathrm{BLR}}$ increases from $\Delta i=51^{\circ}$ to $55^{\circ}$, which indicates that relativistic Doppler boosting effect is weakened compared to the aligned case. Figure 6 illustrates the geometry of the BLR and BBH system, which shows that the BLR, with an opening angle of $\theta_{o}=43^{\circ}$ and viewed by an inclination angle of $i_{\mathrm{BLR}}=33^{\circ}$, is misaligned with the BBH orbital plane by $\Delta i=51^{\circ}$.

The misalignment between the $\mathrm{BBH}$ orbital plane and the circumbinary BLR may explain the apparent contradiction between the periodical variation of optical and UV light curves due to the orbital motion of close to edge-on viewed BBH and the Gaussian profiles of broad emission lines from flattened BLR viewed at an orientation close to face-on (see bottom panels of Fig. 2). Although the optical and UV continua from the BBHs system vary significantly and periodically due to the relativistic 

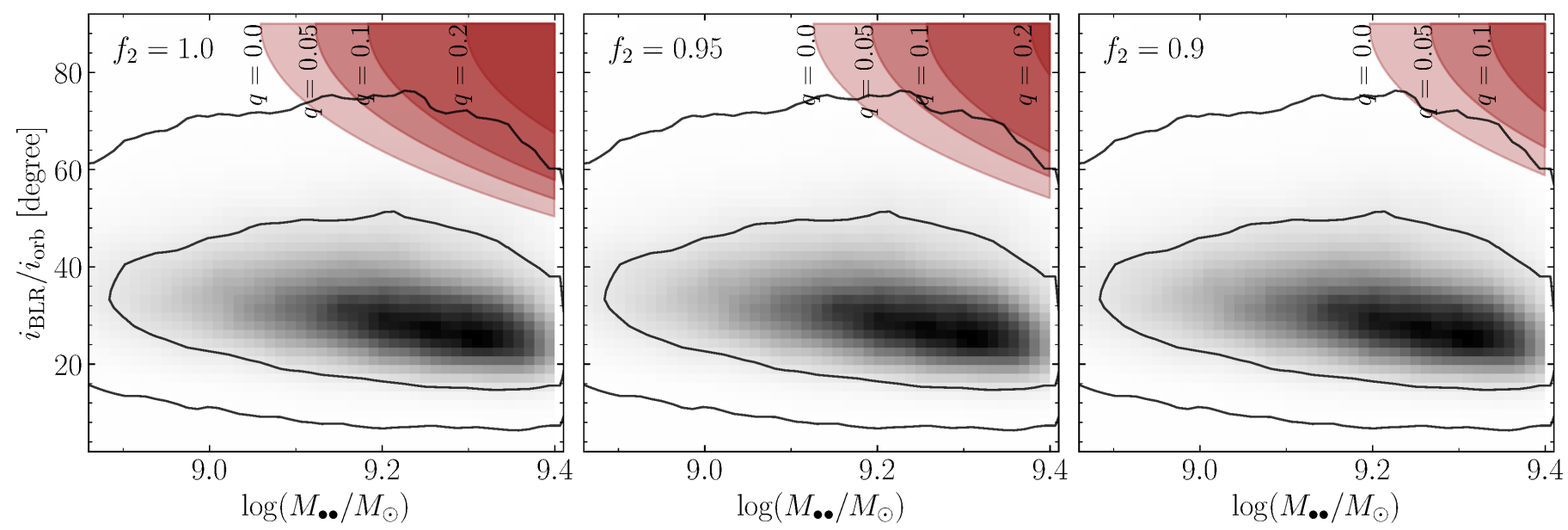

Fig. 4. Posterior PDFs for the total mass of $\mathrm{BBH}\left(M_{\text {... }}\right.$ ) as a function of $i_{\mathrm{BLR}}$ (black) and $i_{\text {orb }}$ (red). Panels show different luminosity fractions of the secondary BH with $f_{2}=100 \%$ (left), 95\% (middle), and 90\% (right) assumed in the Doppler boosting hypothesis (D'Orazio et al. 2015). In each panel, the black shaded region with the $68.3 \%$ and $95.4 \%$ confidence levels overlapped by black solid lines show the PDF result $\left(p\left(i_{\mathrm{BLR}}, M_{\bullet \bullet}\right)\right)$ obtained from stacking the PDFs of the $\mathrm{Ly} \alpha+\mathrm{N}$ v, $\mathrm{C}_{\mathrm{IV}}$, and $\left.\mathrm{C}_{\text {III }}\right]$ broad emission lines with $S / N>3$. As a comparison, the top right shaded region correspond to mass ratios $q=0.0,0.05,0.1$, and 0.2 (from pink to dark red).

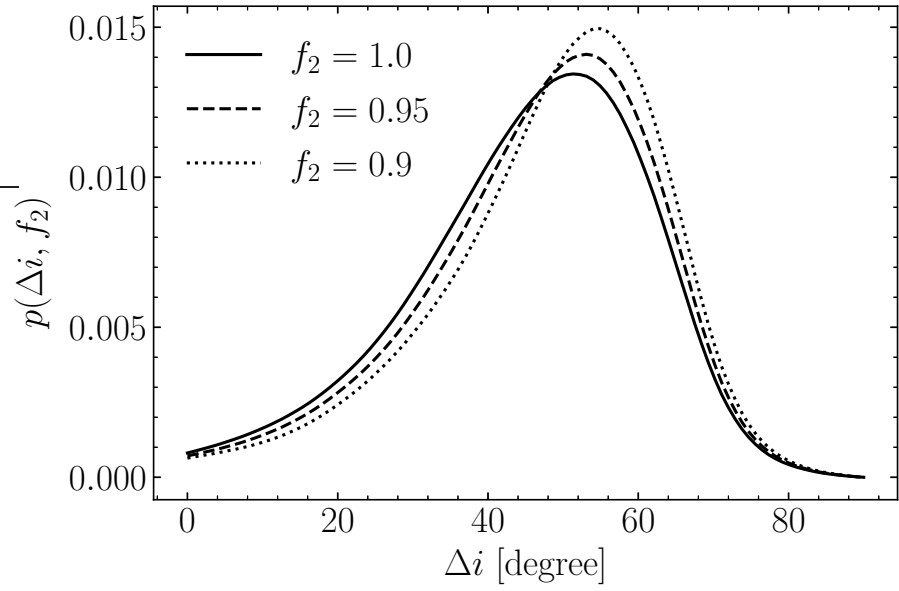

Fig. 5. Probability distribution of the offset between the orientations of the BLR and $\mathrm{BBH}\left(\Delta i=i_{\mathrm{orb}}-i_{\mathrm{BLR}}\right)$ assuming a flat distribution of the BBH mass ratio, the total probabilities are normalized to 1 . Solid, dashed, and dotted lines show the cases with a light contribution from the disk around the secondary $\mathrm{BH}$ of $100 \%, 95 \%$, and $90 \%$, respectively. The estimated peak values of the three probability distributions correspond to $\Delta i=51^{\circ}, 53^{\circ}$, and $55^{\circ}$ for $f_{2}=100 \%, 95 \%$, and $90 \%$, respectively.

Doppler boosting, the ionizing fluxes received by the most of the BLR clouds do not get significantly boosted by the relative motion between the continuum source and the clouds if the BLR middle plane is offset from the BBH orbital plane. Therefore, the Doppler boosting effect on the broad-line emission line profiles is insignificant, and the line variation is much weaker compared with the continuum evolution. It requires much higher spectral $\mathrm{S} / \mathrm{N}$ than the current values to identify the Doppler boosting modulated broad-line profile variations.

\section{Discussions}

The offset of the BLR middle plane from the BBH orbital plane may encode some information about the merger configuration of

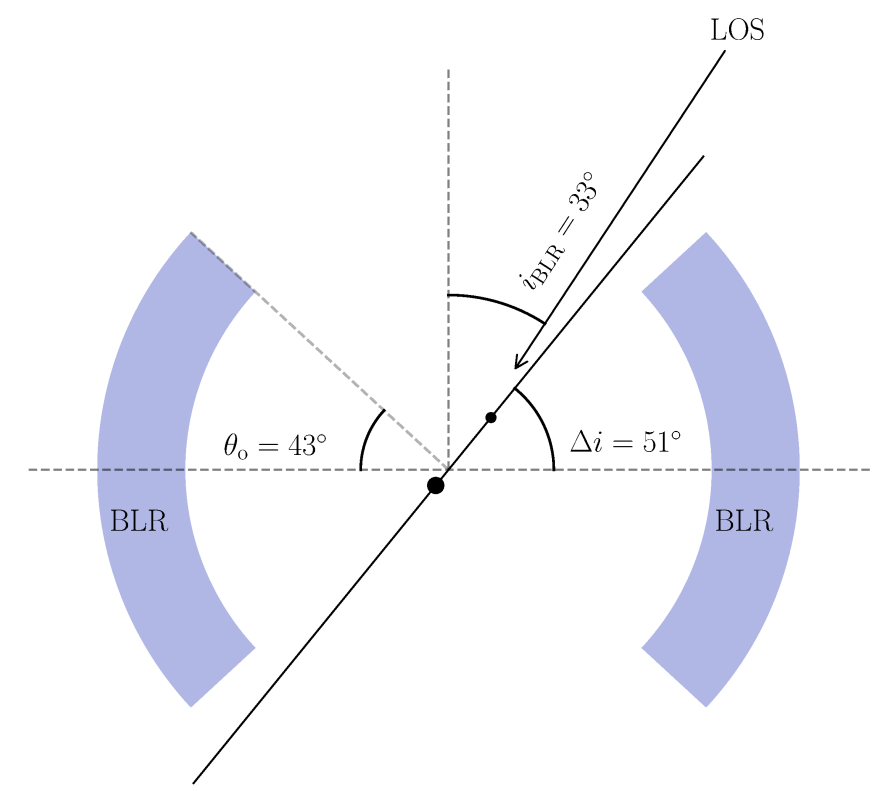

Fig. 6. Cartoon to illustrate the geometry of a BLR offset from the BBH orbital plane as a possible interpretation for both the broad emission line profiles and periodicity of PG 1302-102 under the Doppler boosting scenario. The inclination angle of circumbinary BLR is $\sim 33^{\circ}$, with an opening angle of $\sim 43^{\circ}$. The BBH orbital plane (solid line) is misaligned from the the middle plane of the BLR with an offset angle of $\sim 51^{\circ}$, which indicates a close to edge-on inclination.

the two progenitor galaxies and their nuclear activities. In principle, the two BH components approach each other at a random orientation, and the merger configuration of the two systems that spiral toward each other could be one of the following: 1) two comparable BHs, each having a disk and a BLR ${ }^{7}$; 2) two comparable BHs, one having a disk and a BLR, but the other not; 3) a small $\mathrm{BH}$ and a big $\mathrm{BH}$, both having a disk and a BLR; 4) only the small secondary BH having a disk and a BLR. For

\footnotetext{
7 Here and throughout the middle plane of each progenitor BLR is assumed to be the same as the disk plane.
} 
each of these cases, when the two BHs become close enough, the system may form a (warped or misaligned) circumbinary disk with the central region cultivated to be a hole or gap by the $\mathrm{BBH}$, within the hole or gap each $\mathrm{BH}$ component may still have a small accretion disk surrounding it, and the size of the hole or gap in the case that the two systems merge in a direction inclined to their disk plane is smaller than in their disk plane (e.g., D'Orazio et al. 2013; Farris et al. 2014; Muñoz et al. 2020). In the cases where the two BHs have significantly different masses, the disk around the secondary BH may dominate the total luminosity of the quasi-stellar objects (QSOs) (see, e.g., Graham et al. 2015a; Yan et al. 2015; Charisi et al. 2016). Therefore, the disk orientations would also encode some information about the mergers, but this is beyond the scope of this paper.

For the above four cases, the BLR structure evolves with the orbital decay of the $\mathrm{BBH}$, and the resulting BLR structures from different cases when the BBH system becomes PG1302-102-like may be significantly different. For the first case, the two original BLRs may mix with each other to form a circumbinary BLR, but the initial kinematic structures may be still maintained and thus the profiles of broad emission lines from such a system may be complicated (e.g., with multiple peaks). For the second and fourth cases the resulting system would have a circumbinary BLR with the middle plane offset from the BBH orbital plane as the two systems normally merge in a direction not on the disk plane, and these two planes can be the same only if the merger direction is roughly on the $\mathrm{BBH}$ orbital plane. There may be some differences between the BLR structure resulting from these three cases, but it needs carefully designed simulations to reveal it, which is beyond the scope of this paper. In the third case, the middle plane of the resulting circumbinary BLR would be mainly contributed by the components from the original BLR of the big $\mathrm{BH}$ and it may also be normally offset from the BBH orbital plane; the contribution from the original BLR components of the small $\mathrm{BH}$ are expected to have some small effects on the broad-line profiles and its significance may depend on the relative number of BLR clouds in the two original BLRs. With these cases in mind, we can use the geometric configurations of the BLR in the BBH systems to (statistically) constrain the merger configuration of their progenitor systems.

The significant offset between the BLR middle plane and the BBH orbital plane found for PG1302-102 in this paper, assuming the Doppler boosting scenario, indicates that the configuration of the two progenitor $\mathrm{BH}$ systems is unlikely to be the first case, but it can be the other three cases with two BHs inspiraling in a direction highly inclined to the disk plane of the big $\mathrm{BH}$ in the second and third cases, and to the disk plane of the small $\mathrm{BH}$ in the third case. Future observations and their comparison with detailed simulation results on the BLR configuration would help to test the Doppler boosting hypothesis for optical periodicity of some QSOs and extract important information on the merger configuration of the BBH systems.

The derived offset between the BLR middle plane and the $\mathrm{BBH}$ orbital plane is based on the Doppler boosting assumption, which can produce persistent periodicity in the light curves. However, the optical-UV light curves of PG 1302-102 may be also alternatively explained as being due to (1) a small chance out of a large sample of Quasars with variability due to the DRW processes (e.g., Vaughan et al. 2016), (2) the combination of the DRW and periodic variations or the combination of the variation due to a broken power-law power spectrum and the periodical variation (Liu et al. 2018; Kelly et al. 2009), or (3) the cold-spot in the accretion disk around the more massive black hole of the supermassive BBH system (Kovačević et al. 2019). For these alternative interpretations, the response of the broad emission lines to the continuum variation and the geometry of the BLR deserves further investigations, which is beyond the scope of the present paper.

\section{Conclusions}

The most intriguing BBH candidate among those suggested by the optical periodicity is PG 1302-102, which can be fit well by a model of compact unequal-mass binary system under the relativistic Doppler boosting hypothesis (D'Orazio et al. 2015). In this paper we investigated the properties of broad emission lines of PG 1302-102 using the archive spectroscopic data, including the shapes and variations of $\mathrm{Ly} \alpha+\mathrm{Nv}, \mathrm{C}$ IV , and $\left.\mathrm{C}_{\mathrm{III}}\right]$ lines, and the corresponding parameters of the BLR in order to check whether it is compatible with the Doppler boosting hypothesis. The available observations show that the $\mathrm{Ly} \alpha, \mathrm{C}_{\mathrm{IV}}$, and $\left.\mathrm{C}_{\mathrm{III}}\right]$ all have Gaussian profiles, and the multiple observations of these lines do not suggest significant variations of their profiles, even though the variations, if any, may be smeared out due to the low $\mathrm{S} / \mathrm{Ns}$ and/or spectral resolutions of these observations. Using a simple BLR model we find that the Gaussian profiles of these broad emission lines suggest that the BLR is flattened with an opening angle of $\sim 43^{\circ}$ and viewed at an inclination angle of $\sim 33^{\circ}$, close to face-on, and thus not aligned with the BBH orbital plane, which is viewed close to edge-on, constrained by the optical and UV periodical variations. If the middle plane of the BLR is offset from the BBH orbital plane by an angle of $\sim 51^{\circ}-55^{\circ}$, the periodical optical-UV variations and the broad emission line profiles of PG 1302-102 can be self-consistently explained under the Doppler boosting scenario. In this case the misalignment between the BLR middle plane and the BBH orbital plane leads to a highly weakened Doppler boosting effect on the ionizing flux received by the BLR clouds, thus the variations of emission lines are also much weaker than the continuum received by the observer. This may be taken as a signature to falsify the Doppler boosting scenario for interpreting the periodical optical-UV variations of PG 1302-102 by using multiple high $\mathrm{S} / \mathrm{Ns}$ and high resolution spectroscopic observations in the future.

\section{Acknowledgements}

This work is supported by the National Key Program for Science and Technology Research and Development (Grant No. 2016YFA0400704), the National Natural Science Foundation of China (Grant number 11690024, 11873056 and 11903046), the Strategic Priority Program of the Chinese Academy of Sciences (Grant No. XDB 23040100), and by the Beijing Natural Science Foundation (No. 1204038).

\section{References}

Begelman, M. C., Blandford, R. D., \& Rees, M. J. 1980, Nature, 287, 307

Bentz, M. C., Denney, K. D., Grier, C. J., et al. 2013, ApJ, 767, 149 Boroson, T. A. \& Green, R. F. 1992, ApJS, 80, 109

Charisi, M., Bartos, I., Haiman, Z., et al. 2016, MNRAS, 463, 2145

Charisi, M., Haiman, Z., Schiminovich, D., \& D’Orazio, D. J. 2018, MNRAS, 476, 4617

Chen, Y., Yu, Q., \& Lu, Y. 2020, ApJ, 897, 86

Chen, Y.-C., Liu, X., Liao, W.-T., et al. 2020, arXiv:2008.12329

Cole, S., Lacey, C. G., Baugh, C. M., et al. 2000, MNRAS, 319, 168

D'Orazio, D. J., Haiman, Z. \& MacFadyen, A. 2013, MNRAS, 436, 2997

D’Orazio, D. J., Haiman, Z., \& Schiminovich, D. 2015, Nature, 525, 351 
Eracleous, M., Boroson, T. A., Halpern, J. P., et al. 2012, ApJS, 201, 23

Farris, B. D., Duffell, P., MacFadyen, A. I., et al. 2014, ApJ, 783, 134

Foreman-Mackey, D., Hogg, D. W., Lang, D., et al. 2013, PASP, 125, 306

Graham, M. J., Djorgovski, S. G., Stern, D., et al. 2015, Nature, 518, 74

Graham, M. J., Djorgovski, S. G., Stern, D., et al. 2015, MNRAS, 453, 1562

Gravity Collaboration, Sturm, E., Dexter, J., et al. 2018, Nature, 563, 657

Guo, H., Liu, X., Shen, Y., et al. 2019, MNRAS, 482, 3288

Haehnelt, M. G., \& Kauffmann, G. 2002, MNRAS, 336, L61

Ji, X., Ge, J., Lu, Y., Yan, C., \& Song, Z. 2020, in preparation

Ju, W., Greene, J. E., Rafikov, R. R., et al. 2013, ApJ, 777, 44

Kelly, B. C., Bechtold, J., \& Siemiginowska, A. 2009, ApJ, 698, 895

Korista, K. T. \& Goad, M. R. 2000, ApJ, 536, 284

Kormendy, J., \& Ho, L. C. 2013, ARA\&A, 51, 511

Kovačević, A. B., Popović, L. Č., Simić, S., et al. 2019, ApJ, 871, 32

Li, Y.-R., Wang, J.-M., Zhang, Z.-X., et al. 2019, ApJS, 241, 33

Liao, W.-T., Chen, Y.-C., Liu, X., et al. 2020, arXiv:2008.12317

Liu, X., Shen, Y., Bian, F., et al. 2014, ApJ, 789, 140

Liu, T., Gezari, S., \& Miller, M. C. 2018, ApJ, 859, L12

Liu, T., Gezari, S., Ayers, M., et al. 2019, ApJ, 884, 36

MacLeod, C. L., Ivezić, Z., Kochanek, C. S., et al. 2010, ApJ, 721, 1014

Magorrian, J., Tremaine, S., Richstone, D., et al. 1998, AJ, 115, 2285

Muñoz, D. J., Lai, D., Kratter, K., et al. 2020, ApJ, 889, 114

Nguyen, K. \& Bogdanović, T. 2016, ApJ, 828, 68

Nguyen, K., Bogdanović, T., Runnoe, J. C., et al. 2019, ApJ, 870, 16

Pancoast, A., Brewer, B. J., \& Treu, T. 2014a, MNRAS, 445, 3055

Pancoast, A., Brewer, B. J., Treu, T., et al. 2014, MNRAS, 445, 3073

Ryan, G., \& MacFadyen, A. 2017, ApJ, 835, 199

Savić, D., Marin, F., \& Popović, L. Č. 2019, A\&A, 623, A56

Shen, Y., Richards, G. T., Strauss, M. A., et al. 2011, ApJS, 194, 45

Sigut, T. A. A., \& Pradhan, A. K. 2003, ApJS, 145, 15

Sigut, T. A. A., Pradhan, A. K., \& Nahar, S. N. 2004, ApJ, 611, 81

Somerville, R. S. \& Davé, R. 2015, ARA\&A, 53, 51

Song, Z., Ge, J., Lu, Y., et al. 2020, MNRAS, 491, 4023

Tang, Y., MacFadyen, A., \& Haiman, Z. 2017, MNRAS, 469, 4258

Torrealba, J., Chavushyan, V., Cruz-González, I., et al. 2012, Rev. Mexicana Astron. Astrofis., 48, 9

Tsalmantza, P., Decarli, R., Dotti, M., et al. 2011, ApJ, 738, 20

Vaughan, S., Uttley, P., Markowitz, A. G., et al. 2016, MNRAS, 461, 3145

Vestergaard, M. \& Peterson, B. M. 2006, ApJ, 641, 689

Volonteri, M., Madau, P., \& Haardt, F. 2003, ApJ, 593, 661

Xin, C., Charisi, M., Haiman, Z., et al. 2020, MNRAS, 496, 1683

Yan, C.-S., Lu, Y., Dai, X., \& Yu, Q. 2015, ApJ, 809, 117

Yu, Q. 2002, MNRAS, 331, 935

Zheng, Z.-Y., Butler, N. R., Shen, Y., et al. 2016, ApJ, 827, 56 